\title{
Intramedullary Nailing of Femoral Diaphyseal Metastases: Is it Necessary to Protect the Femoral Neck?
}

\author{
Bryan Moon MD, Patrick Lin MD, Robert Satcher MD, PhD, \\ Justin Bird MD, Valerae Lewis MD
}

Received: 28 May 2014 / Accepted: 12 November 2014/Published online: 26 November 2014

(C) The Association of Bone and Joint Surgeons (B) 2014

\begin{abstract}
Background Intramedullary nailing is the accepted form of treatment for impending or pathologic fractures of the femoral diaphysis. Traditional teaching promotes the use of a cephalomedullary nail so that stabilization is provided for the femoral neck in the event that a future femoral neck metastasis develops. However, that approach may add cost, surgical time, blood loss, and added radiation exposure to staff members, and there is limited evidence in the literature that supports this practice.

Questions/purposes The purpose of our study was to evaluate the incidence of femoral neck metastases in patients who underwent femoral nailing of diaphyseal metastases.

Patients and Methods Retrospective analysis of our Musculoskeletal Oncology database identified 145 femoral nailings performed for metastatic disease, myeloma, or

Each author certifies that he or she, or a member of their immediate family, has no commercial associations (eg, consultancies, stock ownership, equity interest, patent/licensing arrangements, etc) that might pose a conflict of interest in connection with the submitted articles.

All ICMJE Conflict of Interest Forms for authors and Clinical Orthopaedics and Related Research ${ }^{\mathbb{R}}$ editors and board members are on file with the publication and can be viewed on request. Each author certifies that his or her institution approved or waived approval for the human protocol for this investigation and that all investigations were conducted in conformity with ethical principles of research.
\end{abstract}

B. Moon ( $\square)$, P. Lin, R. Satcher, J. Bird, V. Lewis Department of Orthopaedic Oncology, Unit 1448, Anderson Cancer Center, PO Box 301402, Houston, TX 77230, USA

e-mail: bsmoon@mdanderson.org lymphoma of the femoral diaphysis between 2001 and 2011. Average patient age was 59 years. One hundred forty-one patients underwent 145 femoral nailings (four were bilateral). One hundred forty-four of the nails used were cephalomedullary implants and one was a flexible nail. Thirty-six $(25 \%)$ femurs had sustained a pathologic fracture and 109 (75\%) femurs were treated as impending fractures. Eighty-four patients received either preoperative or postoperative radiation therapy. Average radiographic followup was 13 months and average postoperative survival was 16 months. Of the 141 patients in this series, 121 $(86 \%)$ are known to have died at a median of 9 months (range, 0.1-133 months) after surgery. The latest followup radiographs were obtained at a median of 5 months after the femoral nailing (range, 0-119 months). Of the 90 patients with documented dates of death and radiographic followup greater than zero months, 76 (84\%) had radiographs available within a year of death. Thirty-one patients had zero months radiographic followup. The median survival for this group of patients was only 0.9 months (range, 0.1-12 months).

Results No patients $(0 \%)$ in this series had femoral neck metastases develop postoperatively.

Conclusion Despite traditional teaching that supports the use of cephalomedullary implants when treating metastatic disease of the femur, we were unable to identify a single patient who had femoral neck metastasis after surgery on the femur. Our findings do not support the use of cephalomedullary implants in this patient population for the sole purpose of prophylactic femoral neck stabilization; however, this series was relatively small, and the experiences of other centers will be needed to come to a more-complete sense of the frequency of what in all likelihood is a rare event.

Level of Evidence Level IV, therapeutic study. 


\section{Introduction}

Metastatic disease of the femur is a common condition encountered by orthopaedic surgeons. Lesions of sufficient size can weaken the femur and lead to pain, impending fracture, and actual pathologic fractures. These events can cause pain, disability, and worsening of patients' quality of life. Surgery, including prophylactic stabilization or osteosynthesis for femurs that have fractured through metastatic lesions often is indicated.

Intramedullary nailing has become the generally accepted form of treatment for impending and pathologic fractures of the femoral diaphysis [2, 6, 10-12, 17, 18]. Furthermore, traditional teaching has stressed the importance of protecting as much of the femur as possible rather than just the femoral diaphysis. On the basis of this relatively sparse evidence base $[15,16]$, the majority of patients with impending or pathologic fractures are treated with a cephalomedullary nail to protect the femoral neck in the event that a future metastasis develops in the femoral neck [3-5, 7-10, 13-17]. However, using nails that protect the femoral neck in this way has the associated risk of joint penetration, increases operative time and radiation exposure when compared with standard antegrade nails, and to our knowledge, other than the work of van der Hulst et al. [15], there are no data supporting the need to protect the femoral neck in the absence of femoral neck metastasis at the time a femoral shaft metastasis is treated.

The purpose of our study was to determine the incidence of femoral neck metastases in patients with symptomatic, isolated femoral diaphyseal lesions that were treated with surgical stabilization.

\section{Patients and Methods}

A retrospective review of our Musculoskeletal Oncology database identified 293 patients who underwent femoral nailings for metastatic disease between 2001 and 2011. Of the 293 patients, 141 presented with isolated femoral diaphyseal lesions and underwent 145 femoral nailings; four patients had bilateral nailings. Average patient age in our series was 59 years (range, 22-87 years), and the population was comprised of 66 men and 75 women. Diagnoses included 128 patients with metastatic carcinoma, 14 with multiple myeloma, and three with lymphoma (Fig. 1). For the purpose of our study, we defined the femoral diaphysis as the segment of the femur extending from the inferior aspect of the lesser trochanter to the femoral metaphysis. Thirty-six $(25 \%)$ femurs had sustained a pathologic fracture and $109(75 \%)$ were treated as impending fractures. Of the 141 patients with isolated femoral diaphyseal lesions who underwent 145 femoral nailings, 144 instances were treated with a cephalomedullary nail, and one was treated with flexible nails. Eighty-four patients with nailings received either preoperative or postoperative radiation therapy.

Plain radiographs were the primary form of evaluation. The radiographic followup was defined as starting the day of surgery and extending until the last documented image that included imaging of the femoral neck. Patients were not excluded if they died before radiographic followup but rather were assigned a followup of zero months. Average radiographic followup was 13 months (range, 0-119 months). Of the 141 patients in this series, $121(86 \%)$ are known to have died at a median of 9 months (range, 0.1-133 months) after surgery. The latest followup radiographs were obtained at a median of 5 months after the femoral nailing (range, 0-119 months). Of the 90 patients with documented dates of death and radiographic followup greater than zero months, $76(84 \%)$ had radiographs available within a year of death. Thirty-one patients had zero months of radiographic followup. The median survival for this group of patients was only 0.9 months (range, $0.1-12$ months).

Imaging was reviewed by the lead author (BM), a fellowship-trained orthopaedic oncologist, and radiology reports also were reviewed for all imaging studies. There were no instances of conflicting opinion regarding the presence or absence of a femoral neck metastasis.

\section{Results}

No patients $(0 \%)$ in this series had femoral neck metastases postoperatively. One hundred twenty-one patients had documented dates of death. Of these patients, four (3\%) experienced local progression and underwent revision surgery to either an endoprosthesis or intercalary spacer. Three patients (2\%) had failure of the nail and underwent conversion surgery to an endoprosthesis. Two patients (2\%) underwent revision surgery to an arthroplasty owing to the development of metastases in the acetabulum.

\section{Discussion}

Cephalomedullary implants frequently are used for treatment of metastatic disease to the femur with the rationale that the entire length of the bone needs to be protected. While seemingly sensible, support for the practice in the literature is scant $[15,16]$, and the disadvantages of this practice include increased risk of joint penetration, increased surgical time, and radiation exposure to the surgical team members. The increased surgical time and radiation exposure is directly related to the need to adequately see the femoral neck on orthogonal views and verification of placement of screws in the femoral head or neck. Antegrade 


\section{Diagnoses}

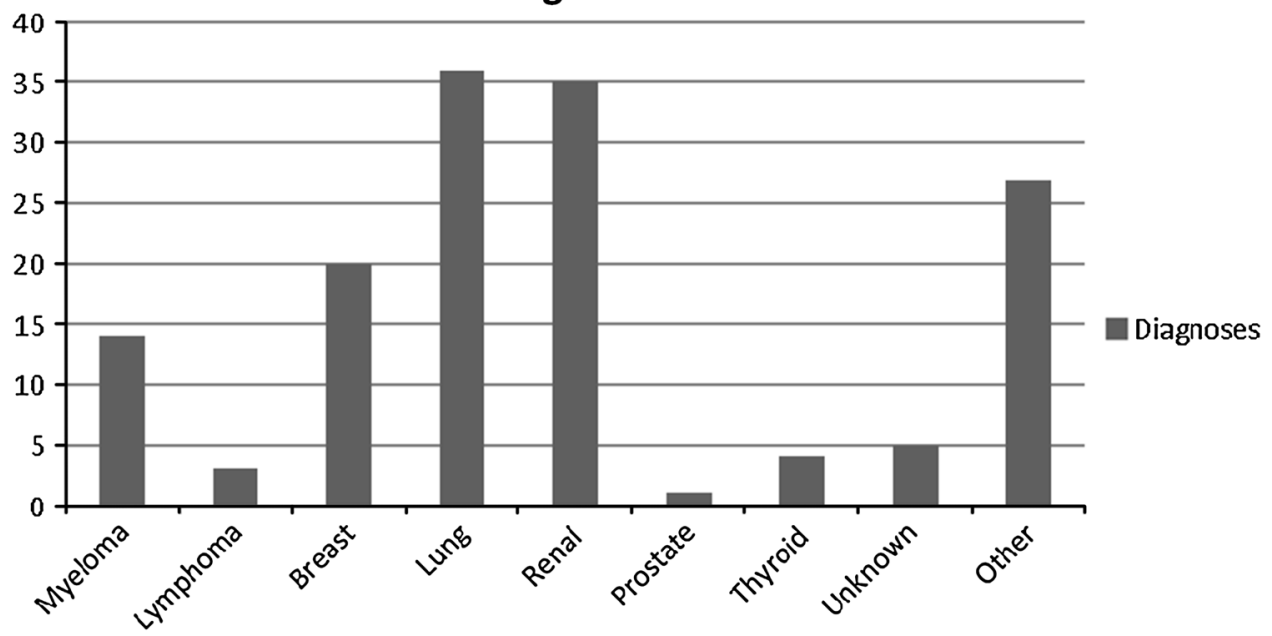

Fig. 1 The cancer diagnoses of our patients are shown. "Other" includes: colon, squamous, penis, esophagus, endometrial, ovarian, gall bladder, liver, melanoma, germ cell, bladder, cervical, and adrenal.

locking does not require this. In addition, there has been some emerging evidence that the entire bone does not require protection $[1,19]$. It is this more recent evidence, combined with the lack of anecdotal support in our practice at a high-volume cancer center that led to a more formal evaluation of our experience, and in this study, we sought to evaluate the incidence of femoral neck metastases in patients who underwent femoral nailing of diaphyseal metastases. Because we teach residents and fellows that it is necessary to protect the entire femur, we wanted to determine whether our teachings were based on actual evidence.

Our study has several limitations, and should be interpreted in light of these. First, not all patients were accounted for; 31 of the 141 patients were lost to radiographic followup; however, the median survival for these patients was only 0.9 months, so the chance that a femoral neck metastasis developed in such a short span of survival is unlikely. Although $84 \%$ of our patients with documented death and greater than zero months followup (90 of 121) had radiographs within a year of death, that still leaves $16 \%$ (31 patients) whose latest radiographs were obtained more than a year before death, and some of these patients may have had femoral neck metastases develop during that time. However, they did not come to us for treatment for this, nor did they become symptomatic that we know of. Second, if we surmise that subsequent femoral neck metastases in patients with isolated diaphyseal metastases are uncommon or rare, it will take more than a database of 141 patients to identify the true frequency of this event. The experiences of other centers will be important to corroborate our experience, although some studies have been published [1, 19] and tend to support our observations. Finally, our selection criteria may have influenced our findings. Our study was limited to patients with isolated diaphyseal metastases. By not including patients with diffuse disease beyond the diaphysis, our findings could be the result of selection bias. Patients with lesions in the intertrochanteric region and lesser trochanter were excluded because an antegrade nail would not provide adequate fixation and typically would not be an appropriate option. An argument could be made that a lesser trochanter lesion could be adequately stabilized with an antegrade nail; however, we did separately evaluate 39 patients with lesser trochanter lesions treated with cephalomedullary nails, and none of them had subsequent femoral neck metastases develop during the study period.

Our findings of zero femoral neck metastases stand in stark contrast to those of van der Hulst et al. [15]. Their study provides the best support for protecting the femoral neck. Their study included 29 patients with impending or pathologic fractures of the femur. Five femoral neck fractures occurred after femoral nailing. From their results, van der Hulst et al. concluded that the entire femur should be stabilized and treated with radiation therapy. However, they also reported that the femoral neck fractures occurred within 5 months of the femoral nailings. Given the short time, they indicated that the femoral neck metastases were likely present at the time of surgery. Although numerous possible explanations for why our results differ from those of van der Hulst et al. may be offered, it seems that a likely one is that since the 1990s when van der Hulst et al. collected the data for their study, better systemic treatment options have been developed, resulting in better control of metastatic disease once it has been identified. This explanation is supported by the fact that average survival of our patient group was 2.5 times longer than that of patients in their study [15]. Another 
explanation is that selection bias had a significant effect on our results. By including only patients with isolated diaphyseal metastases, we were selecting for a specific patient subgroup with femoral metastases. This is important because our results do not imply that femoral neck metastases are rare. We identified and excluded 26 patients who presented with femoral neck metastases at the time of femoral nailing. Rather, our results would indicate that it is rare for a patient who presents with an isolated diaphyseal metastasis to subsequently have femoral neck metastasis develop. Whether these results are attributable to earlier treatment for that particular patient's femoral metastasis or because we selected a subgroup that was destined to not have femoral metastasis develop beyond the diaphysis is impossible to say.

Our findings do not support the use of cephalomedullary implants in this patient population for the sole purpose of prophylactic femoral neck stabilization; however, this series was relatively small, and the experiences of other centers will be needed to come to a more-complete sense of the frequency of what in all likelihood is a rare event. Given our relatively small sample size, our results would not necessarily support a change in practice, but our findings seem to be supported by others on related questions [1, 19]. In addition, we should make the rationale for choice of implant clear to those we train. It is not incorrect to use cephalomedullary nails for femoral diaphyseal metastases, but the surgeon should know what evidence may exist to add the extra surgical time and radiation exposure to prophylactically stabilize the femoral neck in this particular group of patients. These considerations will come into play as we evaluate the costs of various treatment options not covered in this study. Perhaps most importantly, our results reinforce the need for multiinstitutional collaboration, especially in the field of orthopaedic oncology, where the majority of studies have a relatively small number of patients. It is only through this type of collaboration that we will be able to develop treatment standards that are driven by data rather than by historical rationale.

\section{References}

1. Alvi HM, Damron TA. Prophylactic stabilization for bone metastases, myeloma, or lymphoma: do we need to protect the entire bone? Clin Orthop Relat Res. 2013;471:706-714.
2. Arvinius C, Parra JL, Mateo LS, Maroto RG, Borrego AF, Stern LL. Benefits of early intramedullary nailing in femoral metastases. Int Orthop. 2014;38:129-132.

3. Bauer HC. Controversies in the surgical management of skeletal metastases. J Bone Joint Surg Br. 2005;87:608-617.

4. Bickels J, Dadia S, Lidar Z. Surgical management of metastatic bone disease. J Bone Joint Surg Am. 2009;91:1503-1516.

5. Biermann JS, Holt GE, Lewis VO, Schwartz HS, Yaszemski MJ. Metastatic bone disease: diagnosis, evaluation, and treatment. $J$ Bone Joint Surg Am. 2009;91:1518-1530.

6. Giannoudis PV, Bastawrous SS, Bunola JA, Macdonald DA, Smith RM. Unreamed intramedullary nailing for pathological femoral fractures: good results in 30 cases. Acta Orthop Scand. 1999;70:29-32.

7. Gibbons CE, Pope SJ, Murphy JP, Hall AJ. Femoral metastatic fractures treated with intramedullary nailing. Int Orthop. 2000;24: 101-103.

8. Goodman HJ, Patterson FR. Surgical management of lower extremity metastatic disease. In: Biermann JS, ed. Orthopaedic Knowledge Update: Musculoskeletal Tumors 3. Rosemont, IL: American Academy of Orthopaedic Surgeons; 2014:345-353.

9. Harrington KD. Orthopedic surgical management of skeletal complications of malignancy. Cancer. 1997;80:1614-1627.

10. Moholkar K, Mohan R, Grigoris P. The Long Gamma Nail for stabilisation of existing and impending pathological fractures of the femur: an analysis of 48 cases. Acta Orthop Belg. 2004;70: 429-434.

11. Ryan JR, Rowe DE, Salciccioli GG. Prophylactic internal fixation of the femur for neoplastic lesions. J Bone Joint Surg Am. 1976;58: 1071-1074.

12. Samsani SR, Panikkar V, Venu KM, Georgiannos D, Calthorpe D. Breast cancer bone metastasis in femur: surgical considerations and reconstruction with Long Gamma Nail. Eur J Surg Oncol. 2004;30:993-997.

13. Sarahrudi K, Greitbauer M, Platzer P, Hausmann JT, Heinz T, Vecsei V. Surgical treatment of metastatic fractures of the femur: a retrospective analysis of 142 patients. J Trauma. 2009;66:11581163.

14. Swanson KC, Pritchard DJ, Sim FH. Surgical treatment of metastatic disease of the femur. J Am Acad Orthop Surg. 2000;8:5665.

15. van der Hulst RR, van den Wildenberg FA, Vroemen JP, Greve JW. Intramedullary nailing of (impending) pathologic fractures. $J$ Trauma. 1994;36:211-215.

16. Ward WG, Holsenbeck S, Dorey FJ, Spang J, Howe D. Metastatic disease of the femur: surgical treatment. Clin Orthop Relat Res. 2003;415(suppl):S230-244.

17. Weber KL, O'Connor MI. Operative treatment of long bone metastases: focus on the femur. Clin Orthop Relat Res. 2003: 415(suppl)S276-278.

18. Weber KL, Randall RL, Grossman S, Parvizi J. Management of lower-extremity bone metastasis. J Bone Joint Surg Am. 2006; 88(suppl 4):11-19.

19. Xing Z, Moon BS, Satcher RL, Lin PP, Lewis VO. A long femoral stem is not always required in hip arthroplasty for patients with proximal femur metastases. Clin Orthop Relat Res. 2013;471:1622-1627. 